\title{
Objects generated by an arbitrary natural number
}

\author{
Krassimir Atanassov \\ Department of Bioinformatics and Mathematical Modelling, \\ Institute of Biophysics and Biomedical Engineering, \\ Bulgarian Academy of Sciences \\ Acad. G. Bonchev Str., B1. 105, Sofia-1113, Bulgaria \\ Intelligent Systems Laboratory \\ Prof. Asen Zlatarov University, Bourgas-8000, Bulgaria \\ e-mail: krat@bas.bg
}

Received: 4 June $2020 \quad$ Revised: 18 November 2020

Accepted: 20 November 2020

\begin{abstract}
The set $\underline{\operatorname{Set}}(n)$, generated by an arbitrary natural number $n$, is defined. Some arithmetic functions, defined over its elements are introduced. Some of the arithmetic, set-theoretical and algebraic properties of the new objects are studied.
\end{abstract}

Keywords: Algebraic objects, Arithmetic functions, Natural numbers, Sets.

2010 Mathematics Subject Classification: 11A25.

\section{Introduction}

In the present research, some new mathematical objects will be described. They are generated by a fixed arbitrary natural number $n>1$. Let everywhere below it have the canonical form

$$
n=\prod_{i=1}^{k} p_{i}^{\alpha_{i}}
$$

where $k, \alpha_{1}, \alpha_{2}, \ldots, \alpha_{k} \geq 1$ are natural numbers and $p_{1}, p_{2}, \ldots, p_{k}$ are different prime numbers. In [1], the following notations related to $n$ that we will use below, are introduced:

$$
\begin{gathered}
\underline{\operatorname{set}}(n)=\left\{p_{1}, p_{2}, \ldots, p_{k}\right\}, \\
\underline{\operatorname{mult}}(n)=\prod_{i=1}^{k} p_{i} .
\end{gathered}
$$

We will show that these new objects have properties specific to algebra. 


\section{Main definitions}

For the fixed $n \geq 2$, let us define the set

$$
\underline{\operatorname{Set}}(n)=\left\{m \mid m=\prod_{i=1}^{k} p_{i}^{\beta_{i}} \& \mathrm{~h}(n) \leq \beta_{i} \leq \mathrm{H}(n)\right\},
$$

where

$$
\begin{aligned}
& \mathrm{h}(n)=\min \left(\alpha_{1}, \ldots, \alpha_{k}\right), \\
& \mathrm{H}(n)=\max \left(\alpha_{1}, \ldots, \alpha_{k}\right)
\end{aligned}
$$

and let $\omega(n)=k$.

For example, $\underline{\operatorname{Set}}(12)=\underline{\operatorname{Set}}\left(2^{2} .3\right)=\{6,12,18,36\}$ and $\mathrm{h}(12)=1, \mathrm{H}(12)=2, \omega(12)=2$. $\underline{\operatorname{Set}}(72)=\underline{\operatorname{Set}}\left(2^{3} .3^{2}\right)=\{36,72,108,216\}$ and $\mathrm{h}(72)=2, \mathrm{H}(72)=3, \omega(72)=2$.

It is suitable to define

$$
\begin{aligned}
& \underline{\operatorname{Set}}(0)=\{0\} . \\
& \underline{\operatorname{Set}}(1)=\{1\} .
\end{aligned}
$$

Therefore, for each natural number $n, \underline{\operatorname{Set}}(n) \neq \emptyset$.

\section{Properties of $\underline{\operatorname{Set}}(n)$}

We see immediately that for $n$ being a prime number, and more generally, if $n=\underline{\operatorname{mult}}(n)$ and hence, $\mathrm{h}(n)=\mathrm{H}(n)$, then

$$
\underline{\operatorname{Set}}(n)=\{n\} .
$$

Theorem 1. For the natural number $n$ the cardinality $|\underline{\operatorname{Set}}(n)|$ of $\underline{\operatorname{Set}}(n)$ is equal to

$$
|\underline{\operatorname{Set}}(n)|=(\mathrm{H}(n)-\mathrm{h}(n)+1)^{\omega(n)} .
$$

Proof. For $n=\prod_{i=1}^{k} p_{i}^{\alpha_{i}}, \underline{\operatorname{Set}}(n)$ will contain all natural numbers $m$ with $\underline{\text { mult }}(m)=\underline{\text { mult }}(n)$ and with powers between $\mathrm{h}(n)$ and $\mathrm{H}(n)$. Therefore, each $p_{i}$ will be met with $\mathrm{H}(n)-\mathrm{h}(n)+1$ different degrees and this is valid for each of the $\omega(n)$ in number divisors of $n$. Hence, the number of all elements of $\underline{\operatorname{Set}}(n)$ is exactly $(\mathrm{H}(n)-\mathrm{h}(n)+1)^{\omega(n)}$.

For example,

$$
\begin{gathered}
|\underline{\operatorname{Set}}(24)|=\left|\underline{\operatorname{Set}}\left(2^{3} .3\right)\right| \\
=\left|\left\{2.3,2^{2} .3,2^{3} .3,2.3^{2}, 2^{2} .3^{2}, 2^{3} .3^{2}, 2.3^{3}, 2^{2} .3^{3}, 2^{3} .3^{3}\right\}\right| \\
=9=(3-1+1)^{2}, \\
|\underline{\operatorname{Set}}(36)|=\left|\underline{\operatorname{Set}}\left(2^{2} .3^{2}\right)\right|=\left|\left\{2^{2} .3^{2}\right\}\right|=1=(2-2+1)^{2}, \\
|\underline{\operatorname{Set}}(60)|=\left|\underline{\operatorname{Set}}\left(2^{2} .3 .5\right)\right| \\
=\left|\left\{2.3 .5,2^{2} .3 .5,2.3^{2} .5,2^{2} .3^{2} .5,2.3 .5^{2}, 2^{2} .3 .5^{2}, 2.3^{2} .5^{2}, 2^{2} .3^{2} .5^{2}\right\}\right| \\
=8=(2-1+1)^{3} .
\end{gathered}
$$


Theorem 2. For two natural numbers $m$ and $n$, if $m$ is a divisor of $n, \mathrm{~h}(m)=\mathrm{h}(n)$ and $\underline{\text { set }}(m)=$ $\underline{\operatorname{set}}(n)$, then

$$
\underline{\operatorname{Set}}(m) \subseteq \underline{\operatorname{Set}}(n) .
$$

Proof. Having in mind that $m$ is a divisor of $n$, we see that $\mathrm{H}(m) \leq \mathrm{H}(n)$.

Let $t \in \underline{\operatorname{Set}}(m)$. Therefore, $t=\prod_{i=1}^{k} p_{i}^{\gamma_{i}}$, where $\mathrm{h}(m) \leq \gamma_{i} \leq \mathrm{H}(m)$ for each $i=1, \ldots, k$. Hence,

$$
\mathrm{h}(n)=\mathrm{h}(m) \leq \gamma_{i} \leq \mathrm{H}(m) \leq \mathrm{H}(n),
$$

i.e., $t \in \underline{\operatorname{Set}}(n)$.

It is important to note that without one of the conditions $\mathrm{h}(m)=\mathrm{h}(n)$ and $\underline{\operatorname{set}}(m)=\underline{\operatorname{set}}(n)$, the Theorem is not valid. For example, 6 is a divisor of 72 and $\underline{\operatorname{set}}(6)=\underline{\operatorname{set}}(72)=\{2,3\}$, but $\underline{\operatorname{Set}}(6)=\{6\}$, while $\underline{\operatorname{Set}}(72)$ mentioned above, does not contain the element 6 .

On the other hand, 6 is a divisor of 30 and $\mathrm{h}(6)=\mathrm{h}(30)=1$, but $\underline{\operatorname{Set}}(30)=\{30\}$ and hence $\underline{\operatorname{Set}}(6) \nsubseteq \underline{\operatorname{Set}}(30)$.

For the well-known operations "Greatest Common Divisor" and "Least Common Multiple" over two natural numbers $m$ and $n$ that are marked by $(m, n)$ and $[m, n]$, respectively, the following equalities are valid.

Theorem 3. For two natural numbers $m$ and $n$ so that $\underline{\operatorname{set}}(m)=\underline{\operatorname{set}}(n)$ :

$$
\begin{aligned}
& \underline{\operatorname{Set}}(m) \cap \underline{\operatorname{Set}}(n) \subseteq \underline{\operatorname{Set}}((m, n)), \\
& \underline{\operatorname{Set}}(m) \cup \underline{\operatorname{Set}}(n) \supseteq \underline{\operatorname{Set}}([m, n]) .
\end{aligned}
$$

Proof. Let $t \in \underline{\operatorname{Set}}(m) \cap \underline{\operatorname{Set}}(n)$. Therefore, $t=\prod_{i=1}^{k} p_{i}^{\gamma_{i}}$, where $\gamma_{1}, \ldots, \gamma_{k} \geq 1$ are natural numbers. From the fact that $t \in \underline{\operatorname{Set}}(m)$ it follows that $\mathrm{h}(m) \leq \gamma_{i} \leq \mathrm{H}(m)$ and from the fact that $t \in \underline{\operatorname{Set}}(n)$ it follows that $\mathrm{h}(n) \leq \gamma_{i} \leq \mathrm{H}(n)$ for $i=1, \ldots, k$. Therefore

$$
\max (\mathrm{h}(m), \mathrm{h}(n)) \leq \gamma_{i} \leq \min (\mathrm{H}(m), \mathrm{H}(n)) .
$$

Obviously, when $\max (\mathrm{h}(m), \mathrm{h}(n))>\min (\mathrm{H}(m), \mathrm{H}(n))$, the number $t$ does not exist. For example,

$$
\underline{\operatorname{Set}}(6) \cap \underline{\operatorname{Set}}(36)=\{6\} \cap\{36\}=\emptyset .
$$

Therefore, (1) is valid.

Having in mind that

$$
(m, n)=\prod_{i=1}^{k} p_{i}^{\min \left(\alpha_{i}, \beta_{i}\right)}
$$

for $\underline{\operatorname{Set}}((m, n))$ we see that

$$
\underline{\operatorname{Set}}((m, n))=\left\{u \mid u=\prod_{i=1}^{k} p_{i}^{\varepsilon_{i}} \& \min (\mathrm{h}(m), \varepsilon(n)) \leq \delta_{i} \leq \min (\mathrm{H}(m), \mathrm{H}(n))\right\} .
$$

Hence, when $\max (\mathrm{h}(m), \mathrm{h}(n)) \leq \min (\mathrm{H}(m), \mathrm{H}(n))$, for $t$ it is valid that

$$
\min (\mathrm{h}(m), \mathrm{h}(n)) \leq \max (\mathrm{h}(m), \mathrm{h}(n)) \leq \gamma_{i} \leq \min (\mathrm{H}(m), \mathrm{H}(n)),
$$

i.e., $t \in \operatorname{Set}((m, n))$. 
In the opposite case, if $t \in \underline{\operatorname{Set}}((m, n))$, then

$$
\min (\mathrm{h}(m), \mathrm{h}(n)) \leq \gamma_{i} \leq \min (\mathrm{H}(m), \mathrm{H}(n)) .
$$

If $\mathrm{h}(m) \leq \mathrm{h}(n)$, then it will be certain that $t \in \underline{\operatorname{Set}}(m)$, but only if $\mathrm{h}(n) \leq \gamma_{i}$ for each $i=1, \ldots, k$, then $t \in \underline{\operatorname{Set}}(n)$ and therefore, $t \in \underline{\operatorname{Set}}(m) \cap \underline{\operatorname{Set}}(n)$.

Hence (1) is valid. The validity of (2) is proved in the same manner.

\section{Algebraic objects generated by an arbitrary natural number}

Let us define for the fixed $n$ :

$$
\begin{aligned}
& \square n=(\underline{\text { mult }}(n))^{\mathrm{h}(n)}, \\
& \text { ๒ } n=(\underline{\text { mult }}(n))^{\mathrm{H}(n)},
\end{aligned}
$$

and for each $m \in \underline{\operatorname{Set}}(n)$ :

$$
\neg m=\prod_{i=1}^{k} p_{i}^{\mathrm{H}(n)+\mathrm{h}(n)-\beta_{i}} .
$$

We see immediately, that $\square n$, 田 $n \in \underline{\operatorname{Set}}(n)$, and for each $m \in \underline{\operatorname{Set}}(n): \neg m \in \underline{\operatorname{Set}}(n)$. Moreover,

$$
\neg m=\frac{\text { mult }(n)^{\mathrm{H}(n)+\mathrm{h}(n)}}{m}=\frac{\boxminus n . \circledast n}{m} .
$$

Therefore

$$
\begin{aligned}
& \neg @ n=\circledast n, \\
& \neg \circledast n=\emptyset n .
\end{aligned}
$$

Following [3], we will mention that if $S$ is a fixed set with unit element $e_{S}$ and if $*$ is an operation over $S$, then $\left\langle S, *, e_{S}\right\rangle$ is a commutative monoid, if:

1. $(\forall u, v \in S)(u * v \in S)$,

2. $(\forall u, v, w \in S)(u *(v * w)=(u * v) * w)$,

3. $(\forall a \in S)\left(u * e_{S}=u=e_{S} * u\right)$,

4. $(\forall u, v \in S)(u * v=v * u)$.

Now, we prove the following theorem.

Theorem 4. For the fixed $n$ :

(a) $\langle\underline{\operatorname{Set}}(n),()$, 困 $n\rangle$,

(b) $\langle\underline{\operatorname{Set}}(n),[]$, @ $n\rangle$

are commutative monoids. 
Proof. Let $n$ be fixed. To see the validity of (a), we check sequentially the following equalities.

Let $u, v, w \in \underline{\operatorname{Set}}(n)$. Therefore,

$$
u=\prod_{i=1}^{k} p_{i}^{\beta_{i}}, \quad v=\prod_{i=1}^{k} p_{i}^{\gamma_{i}}, \quad w=\prod_{i=1}^{k} p_{i}^{\delta_{i}},
$$

where for each $i=1,2, \ldots, k: \mathrm{h}(n) \leq \beta_{i}, \gamma_{i}, \delta_{i} \leq \mathrm{H}(n)$. Hence,

$$
(u, v)=\prod_{i=1}^{k} p_{i}^{\min \left(\beta_{i}, \gamma_{i}\right)}
$$

and from $\mathrm{h}(n) \leq \min \left(\beta_{i}, \gamma_{i}\right) \leq \mathrm{H}(n)$ it follows that $(u, v) \in \underline{\operatorname{Set}}(n)$.

$$
\begin{aligned}
(u,(v, w)) & =\left(\prod_{i=1}^{k} p_{i}^{\beta_{i}},\left(\prod_{i=1}^{k} p_{i}^{\gamma_{i}}, \prod_{i=1}^{k} p_{i}^{\delta_{i}}\right)\right) \\
& =\left(\prod_{i=1}^{k} p_{i}^{\beta_{i}}, \prod_{i=1}^{k} p_{i}^{\min \left(\gamma_{i}, \delta_{i}\right)}\right) \\
& \left.=\prod_{i=1}^{k} p_{i}^{\min \left(\beta_{i}, \min \left(\gamma_{i}, \delta_{i}\right)\right.}\right)=\prod_{i=1}^{k} p_{i}^{\min \left(\beta_{i}, \gamma_{i}, \delta_{i}\right)}=\prod_{i=1}^{k} p_{i}^{\min \left(\min \left(\beta_{i}, \gamma_{i}\right), \delta_{i}\right)} \\
& =\left(\prod_{i=1}^{k} p_{i}^{\min \left(\beta_{i}, \gamma_{i}\right)}, \prod_{i=1}^{k} p_{i}^{\delta_{i}}\right) \\
& =\left(\left(\prod_{i=1}^{k} p_{i}^{\beta_{i}}, \prod_{i=1}^{k} p_{i}^{\gamma_{i}}\right), \prod_{i=1}^{k} p_{i}^{\delta_{i}}\right) \\
& =((u, v), w) . \\
(u, \circledast(n)) & =\prod_{i=1}^{k} p_{i}^{\min \left(\beta_{i}, \mathrm{H}(n)\right)}=\prod_{i=1}^{k} p_{i}^{\beta_{i}}=u=\prod_{i=1}^{k} p_{i}^{\min \left(\mathrm{H}(n), \beta_{i}\right)}=(\text { 因 }(n), u) . \\
(u, v) & =\prod_{i=1}^{k} p_{i}^{\min \left(\beta_{i}, \gamma_{i}\right)}=\prod_{i=1}^{k} p_{i}^{\min \left(\gamma_{i}, \beta_{i}\right)}=(v, u) .
\end{aligned}
$$

The validity of the second assertion is proved in the same manner.

In [2], the author introduced the following concepts.

We call $\left\langle M, *, e_{*}, e_{\circ}\right\rangle$ a "(commutative) multi unitary group" (shortly, (c-) $\mu$-group) if and only if $e_{0} \in M,\left\langle M, *, e_{*}\right\rangle$ is a (commutative) monoid and

$$
(\forall a \in M)\left(\exists a_{\circ} \in M\right)\left(a * a_{\circ}=e_{\circ}=a_{\circ} * a\right) .
$$

Two (c-) $\mu$-groups $M G_{1}$ and $M G_{2}$ are dual, if and only if they have the forms

$$
M G_{1}=\left\langle M, *, e_{*}, e_{\circ}\right\rangle \text { and } M G_{2}=\left\langle M, \circ, e_{\circ}, e_{*}\right\rangle
$$

for some given operations $*$ and $\circ$, and for the unitary elements $e_{*}, e_{\circ} \in M$.

Theorem 5. For the fixed natural number $n$

$$
\langle\underline{\operatorname{Set}}(n),(.), \circledast n, \oslash n\rangle \text { and }\langle\underline{\operatorname{Set}}(n),[.], \square n, \circledast n\rangle
$$

are dual (c-) $\mu$-groups. 
Proof. From Theorem 4 we saw that $\langle\underline{\operatorname{Set}}(n),($.$) , 目 n\rangle$ and $\langle\underline{\operatorname{Set}}(n),[$.$] , @ n\rangle$ are commutative monoids. Now, we see that for arbitrary $u \in \underline{\operatorname{Set}}(n)$ :

$$
(u, \oslash n)=\oslash n=(\oslash n, n)
$$

and

$$
[u, \circledast n]=\circledast n=[\circledast n, n],
$$

i.e., condition (3) is satisfied and hence $\langle\underline{\operatorname{Set}}(n),(),. \circledast n$, $\square n\rangle$ and $\langle\underline{\operatorname{Set}}(n),[],. \square n, \circledast n\rangle$ are dual (c-) $\mu$-groups.

\section{Conclusion}

In a next research, other properties of the introduced here objects will be discussed. In addition, we will show that these objects have properties specific for modal logic.

\section{References}

[1] Atanassov, K. (1985). Short proof of a hypothesis of A. Mullin. Bulletin of Number Theory and Related Topics, Vol. IX (2), 9-11.

[2] Atanassov, K. (2017). Intuitionistic Fuzzy Logics, Springer, Cham.

[3] MacLane, S., \& Birkhoff, G. (1967). Algebra, The Macmillan Co., New York. 